\section{PEER-REVIEWED ARTICLE}

Food Protection Trends, Vol 41, No. 5, p. 476-484
Toni de Senna, * Shirin J. Abd, Eva Borjas, Anne Nillo, and Carrie Ferstl

Food Safety Research, Eurofins Microbiology Laboratories, Inc. 2241 Constitution Drive, Livermore, CA 94551, USA

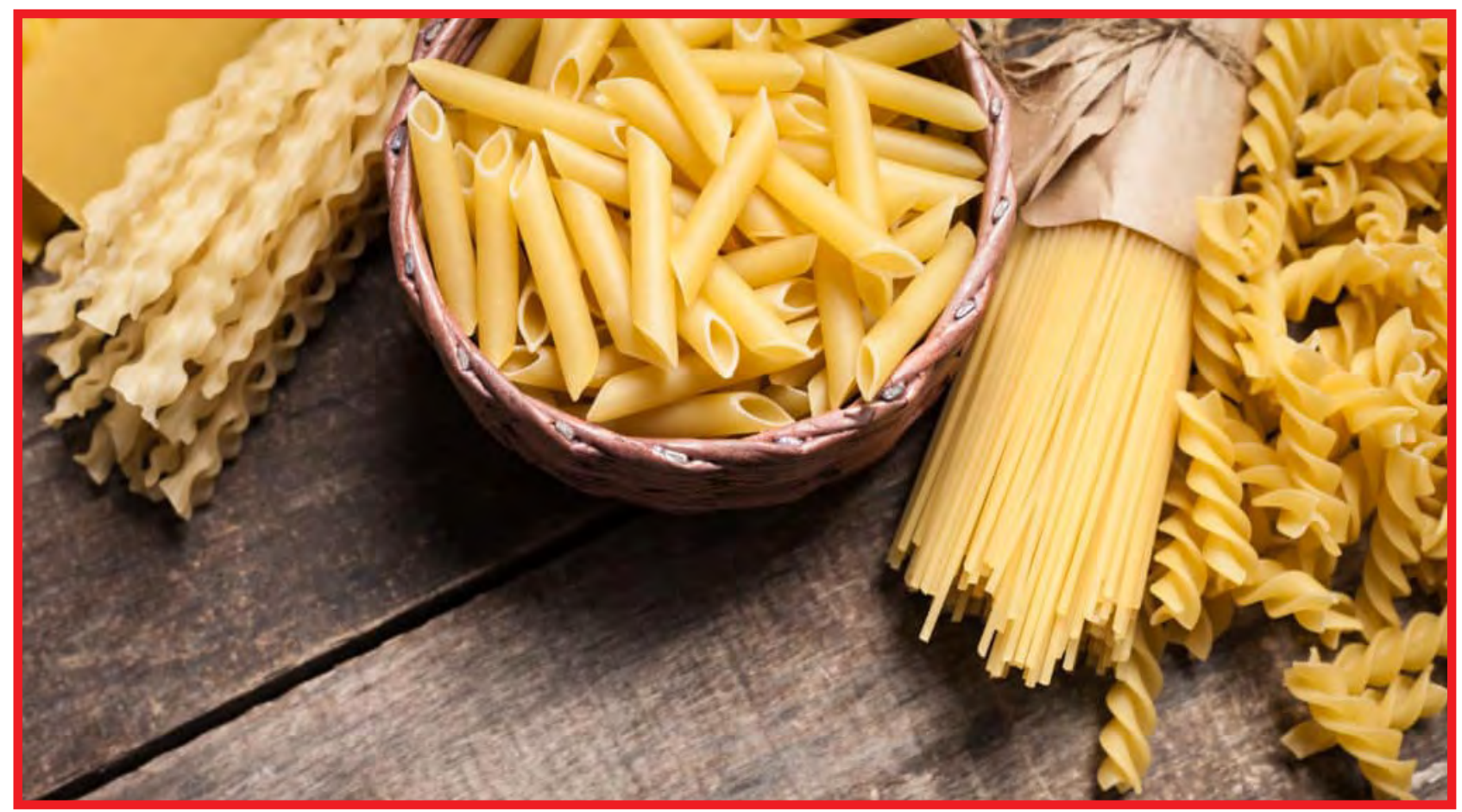

\title{
Reduction of Salmonella enterica and Pathogenic Escherichia coli in Pasta by Using Common Cooking Methods
}

\section{ABSTRAGT}

Recent outbreaks and recalls associated with Salmonella enterica and pathogenic Escherichia coli in flour, a major ingredient in pasta, have prompted assessment of the microbiological lethality of cooking methods used to prepare dry pasta products for consumption. In this study, three types of dry pasta inoculated with multistrain cocktails of $S$. enterica and pathogenic $E$. coli were subjected to typical cooking treatments: elbow noodles were exposed to microwave cooking ( $2.5 \mathrm{~min}$ on high setting; minimum power output of $720 \mathrm{~W}$ ) as part of an "elbow noodles in a cup" product, flat noodles were cooked on a stovetop in boiling water ( 6 min in boiling water), and no-boil lasagna noodles were exposed to baking as part of a three-layer lasagna ( $50 \mathrm{~min}$ in an oven preheated to $204^{\circ} \mathrm{C}$ ). The average population levels of $S$. enterica and pathogenic $E$. coli in all three types of the dry noodles were $>6.5$ log most probable number per gram. Each cooking method evaluated reduced $S$. enterica and pathogenic $E$. coli population levels to $<0.5$ log most probable number per gram, resulting in $>6$-log reductions. The results of this study demonstrate that the cooking processes evaluated are capable of mitigating the microbiological food safety risks associated with dry pasta.

\section{INTRODUCTION}

The potential microbiological food safety risks associated with dry pasta products include Salmonella enterica and pathogenic Escherichia coli. These two pathogens are of concern because of their abilities to cause illness with very low infective doses (31) and survive in low-water-activity products for prolonged periods $(18,23,25)$. Although dry pasta products have not been directly implicated in any foodborne illness outbreaks, outbreaks and recalls associated with a major pasta ingredient (such as flour) or similar low-moisture products have been reported. For example, outbreaks of $S$. enterica have occurred in dry grain-based products such as raw flour, organic sprouted chia powder, and toasted oats cereal $(4-6,20)$, and E. coli O121 and E. coli $\mathrm{O} 26$ have been linked to foodborne illness outbreaks traced to flour $(7,8)$. In addition, possible $S$. enterica contamination has led to recalls in all-purpose flour, brown 
rice flour, and soybean flour $(14,30,32,34)$, as has suspected contamination of flour with pathogenic E. coli $(8,33)$. Thus, the lethality of cooking methods used to prepare dry pasta products for consumption warrants assessment.

One product parameter that may substantially impact the lethality of a cooking method is the water activity of the product. In general, a decrease in the heat resistance of S. enterica and pathogenic E. coli is observed as water activity increases $(1,9,15,24,25,28)$. At low water activities, S. enterica is extremely resistant to heat in general $(13,23)$ and when compared with the heat resistance of pathogenic E. coli (11, 16, 24). Conversely, S. enterica and pathogenic E. coli are typically readily destroyed by heat in high-wateractivity matrices (26). During cooking, dry pasta products become hydrated as they are exposed to heat, transitioning the water activity of the product from low $(<0.70)$ to high $(>0.97)$. The dynamic nature of the water activity of dry pasta as it cooks makes the level of lethality delivered of the cooking process difficult to predict. Therefore, in this study, the fate of $S$. enterica and pathogenic E. coli was determined in representative dry pasta products when exposed to common cooking methods: microwave cooking of elbow noodles, stovetop cooking of flat noodles, and baking of no-boil lasagna noodles as part of lasagna.

\section{MATERIALS AND METHODS}

\section{Pasta ingredients and pasta-making equipment}

Enriched durum flour and whole egg powder were provided by the manufacturer (Zerega's Sons, Inc., Fair Lawn, NJ). Food coloring, low-moisture shredded mozzarella, shredded Parmesan cheese, eggs, and spaghetti sauce were purchased from a local grocery store, and municipal water was used as an ingredient and in the microwave cooking and stovetop cooking methods. The National Pasta Association (NPA; Washington, D.C.) provided elbow noodles, flat noodles, and no-boil lasagna noodles (both noodles that were commercially available and noodles produced exclusively for the study), microwave cups (cup dimensions: $7.62 \mathrm{~cm}$, tall; $7.62 \mathrm{~cm}$, bottom diameter; 10.16 $\mathrm{cm}$, top diameter), and a powdered antifoaming agent.

A stand mixer (KitchenAid ${ }^{\circledR}$, Benton Harbor, MI) was used to combine the pasta ingredients into dough. The NPA provided equipment custom made for the bench-top extrusion and drying of three pasta products: elbow noodles, flat noodles, and no-boil lasagna noodles. The equipment included a pasta press (hand crank), three pasta dies, and a pasta dryer.

\section{Pasta-cooking equipment}

Two 1,100-W microwave ovens were purchased from a local store to deliver the microwave cooking treatment. The actual power output (in Watts) of the microwaves was determined using the International Electrotechnical Commission method IEC 60705 ed. 3.2, 2006. The first microwave (1.1 cu ft, model FFCM1134LS; Frigidaire; Charlotte, NC) was used to treat the S. enterica-inoculated elbow noodles and had an average power output of $720 \pm 25$ $\mathrm{W}(n=6$; data not shown). The second microwave $(1.1 \mathrm{cu} \mathrm{ft}$, model OGZJ1104; Oster, Boca Raton, FL) was used to treat the pathogenic $E$. coli-inoculated elbow noodles and had an average power output of $736 \pm 3 \mathrm{~W}$ ( $n=3$; data not shown).

An electric four-burner $\mathrm{GE}^{\circledR}$ range (model JB700SJ2SS; General Electric, Louisville, KY) was used to deliver the stovetop cooking treatment and the baking treatment to the flat noodles and no-boil lasagna noodles, respectively.

\section{Pathogenic organisms and preparation of inoculum cocktails}

In total, four strains of $S$. enterica and five strains of pathogenic E. coli were included in the study. S. enterica serotypes Agona (ATCC BAA 707; isolation: plant, 1996), Typhimurium (ATCC 13311; isolation: food poisoning, feces, human, 1911), Senftenberg (ATCC 43845; 775W; isolation: no information provided), and Tennessee (ATCC 10722; isolation: no information provided); and E. coli O104:H4 (ATCC BAA 2309; isolation: human stool, Republic of Georgia, patient exhibited hemolytic uremic syndrome, May 2011) and E. coli O111:H8 (ATCC BAA 184; isolation: diarrheal human stool, South Dakota, 2000) were obtained from the American Type Culture Collection (Manassas, VA). E. coli O121 (NFPA 4226; isolation: flour/flour-based outbreak, 2016) and two strains of E. coli O157:H7 (NFPA 4200 isolation: hamburger isolate/Jack-in-the-Box outbreak and NFPA 4217, USDA RM1913; isolation: patient isolate, lettuce outbreak) were obtained from the Eurofins Culture Collection (Fresno, CA). All strains were maintained as frozen stock at $-70^{\circ} \mathrm{C}$ for long-term storage.

Multistrain cocktails of S. enterica and pathogenic E. coli were prepared according to a method adapted from Danyluk et al. (10) and Blessington et al. (3). In brief, each strain was streaked from frozen stock onto tryptic soy agar (TSA; Accumedia, Lansing, MI) and incubated at $35^{\circ} \mathrm{C}$ for 24 to 48 h. An isolated colony of each strain was individually cultured into tryptic soy broth (Accumedia) and incubated at $35^{\circ} \mathrm{C}$ for 18 to $24 \mathrm{~h}$. Aliquots of each culture were then spread onto large prepoured TSA plates and incubated at $35^{\circ} \mathrm{C}$ for 24 $\pm 2 \mathrm{~h}$. After incubation, the cell lawns were harvested using a sterile spreader and $0.1 \%$ peptone water (PW; Difco, BD, Franklin Lakes, NJ). Harvested cells were then combined in equal volumes by organism to produce two multistrain inoculum cocktails - one of S. enterica and one of pathogenic E. coli. Each inoculum cocktail was then combined with an equivalent volume of sterile water and an aliquot of food coloring (added to facilitate the differentiation between inoculated and uninoculated noodles).

\section{Preparation of inoculated dry pasta}

Three types of inoculated dry pasta-elbow noodles, flat noodles, and no-boil lasagna noodles - were produced for the study. The methods for the preparation of each type of 
pasta were provided by the NPA (Table 1). In brief, the dry and wet ingredients were combined and formed into dough by using the stand mixer, and then the dough was allowed to rest under refrigeration conditions for at least $40 \mathrm{~min}$. After resting, the dough was extruded through the appropriate pasta die by using the pasta press; the extruded dough was cut to a suitable noodle length as it exited the pasta die with a sanitized knife. The noodles were then placed in the pasta dryer and allowed to dry to a target water activity of 0.55 to 0.65 (approximately 18 to $24 \mathrm{~h}$ ). Once drying was complete, the inoculated dry pasta noodles were packaged by type in sterile plastic bags and stored under ambient conditions until used in the study.

One batch of $S$. enterica-inoculated noodles per noodle type was prepared for the study. The elbow noodles and flat noodles inoculated with pathogenic $E$. coli were produced in one batch each, and the pathogenic E. coli-inoculated no-boil lasagna noodles were prepared in two independent batches.

\section{Microwave cooking of elbow noodles}

The inoculated elbow noodles were exposed to the microwave cooking treatment as part of an "elbow noodles in a cup" product. The parameters of the microwave cooking treatment were selected to represent handling typical of an elbow noodles in a cup product when being prepared for consumption.

In total, five inoculated batches of elbow noodles per pathogenic organism were subjected to the microwave cooking treatment; each batch was prepared immediately before use. To make an inoculated batch, $6 \mathrm{~g}$ of inoculated elbow noodles and $30 \mathrm{~g}$ of uninoculated elbow noodles were placed into one cooking cup. Next, $3.8 \mathrm{~g}$ of antifoaming agent and $150 \mathrm{~g}$ of warm water $\left(32 \pm 1^{\circ} \mathrm{C}\right)$ were added to the cup, and the total contents of the cup were stirred for $30 \mathrm{~s}$. Each batch was then individually microwaved for $2.5 \mathrm{~min}$ on high setting. Immediately after the microwave cooking treatment was applied, the batch was removed from the microwave and transferred to a sterile plastic bag. The contents of the bag were submerged in an ice water bath for $20 \mathrm{~min}$ to cool the batch quickly and arrest further heat treatment. Once cool, the inoculated elbow noodles were collected using sterile forceps and transferred to a sterile plastic bag; each aliquot of inoculated noodles was considered to be one sample. In addition, two uninoculated batches were prepared per pathogenic organism by using uninoculated elbow noodles and treated in the same manner as the inoculated batches.

In total, three positive control samples per pathogenic organism were prepared. To make a positive control sample, $6 \mathrm{~g}$ of dry inoculated elbow noodles was transferred to a sterile plastic bag and diluted 1:10 (by weight) with PW. The bag was then submerged in an ice water bath for $20 \mathrm{~min}$ to mimic the handling of the microwave-treated samples.

The water activity of the inoculated elbow noodles was measured immediately before inclusion into the elbow noo- dles in a cup product and immediately after the application of the microwave cooking treatment. The temperature of each inoculated batch was also measured immediately before and immediately after exposure to the microwave treatment by using an Omega HH84 rugged temperature data logger (OMEGA Engineering Inc.) and $\mathrm{K}$ type thermocouple (SA1XL-K-120-SRTC, OMEGA Engineering Inc.). The experiment was executed twice per pathogenic organism.

\section{Stovetop cooking of flat noodles}

The inoculated flat noodles were exposed to the stovetop cooking treatment as part of a 100-g batch of flat noodles in boiling water. The parameters of the stovetop cooking treatment were selected to represent handling typical of a flatnoodle type product when being prepared for consumption.

One inoculated batch of flat noodles per pathogenic organism was subjected to the stovetop cooking treatment; each batch was prepared immediately before use. To make an inoculated batch, 25 to $50 \mathrm{~g}$ of inoculated flat noodles was mixed with enough uninoculated flat noodles to yield a final batch mass of $100 \mathrm{~g}$. Next, a total of $0.833 \mathrm{~L}$ of water was brought to a rolling boil on the stovetop, and the batch of flat noodles was added to the boiling water and allowed to cook for $6 \mathrm{~min}$ uncovered. After 6 min of cooking, the flat noodles were separated from the water with a colander and then were transferred to a sterile plastic bag. The batch was submerged in an ice water bath for $20 \mathrm{~min}$ to cool the flat noodles quickly and arrest further heat treatment. After cooling, sterile forceps were used to collect the inoculated flat noodles and divide them into five equal aliquots (by weight) into sterile plastic bags; each aliquot of inoculated noodles was considered to be one sample. In addition, one uninoculated batch per pathogenic organism was prepared using uninoculated flat noodles and treated in the same manner as the inoculated batch; two replicate samples were collected from each uninoculated batch.

In total, three positive control samples per pathogenic organism were prepared. To make a positive control sample, 5 to $10 \mathrm{~g}$ of dry inoculated flat noodles was transferred to a sterile plastic bag and diluted 1:10 dilution (by weight) with PW. The bag was then submerged in an ice water bath for 20 min to mimic the handling of the stovetop-treated samples.

The water activity of the inoculated flat noodles was measured immediately before inclusion into the batch and immediately after the application of the stovetop cooking treatment. The experiment was executed twice per pathogenic organism.

\section{Baking cooking of no-boil lasagna noodles}

The inoculated no-boil lasagna noodles were exposed to the baking cooking treatment as part of a three-layer lasagna. The parameters of the baking cooking treatment were selected to represent handling typical of a no-boil lasagna noodle product when being prepared for consumption. The 


\section{TABLE 1. Recipes for the three pasta types-elbow noodles, flat noodles, and no-boil}

lasagna noodles-provided by the National Pasta Association

\begin{tabular}{c|c|c|c}
\multirow{2}{*}{ Ingredient } & \multicolumn{2}{|c}{ Amount of each ingredient for each pasta type (g) } \\
\cline { 2 - 4 } & Elbow noodles & Flat noodles & No-boil lasagna noodles \\
\hline Enriched durum flour & 200 & 200 & 300 \\
\hline Whole egg powder & 12 & 12 & 18 \\
\hline Water $^{a}$ & 75 & 75 & 113 \\
\hline
\end{tabular}

${ }^{a}$ The water was substituted for an inoculum cocktail when preparing the inoculated pastas.

\section{TABLE 2. Lasagna recipe provided by the National Pasta Association}

Lasagna ingredients and materials

\begin{tabular}{c|c}
\hline $15 \mathrm{oz}$ of Ricotta cheese & $26 \mathrm{oz}$ of spaghetti sauce \\
\hline 1 cup of low-moisture Mozzarella cheese, shredded & 1 cup of water \\
\hline $1 / 2$ cup of Parmesan cheese, grated & 12 no-boil lasagna noodles \\
\hline 1 egg & 1 glass baking dish $(13$ by 9 by 2 in. $)$ \\
\hline
\end{tabular}

Lasagna assembly procedure

Combine $15 \mathrm{oz}$ of Ricotta cheese, 0.5 cup of Mozzarella cheese, 0.25 cup of Parmesan cheese, and 1 egg

Combine 26 oz of spaghetti sauce and 1 cup of water

Spread 1.5 cups of the sauce-water mixture over the bottom of the baking dish

Place four no-boil lasagna noodles atop the sauce-water mixture in a single layer

Spread half of the cheese-egg mixture atop the no-boil lasagna noodles

Spread a third of the remaining sauce-water mixture atop the cheese-egg mixture

Place four no-boil lasagna noodles atop the sauce-water mixture in a single layer

Spread remaining cheese-egg mixture atop the no-boil lasagna noodles

Spread half of the remaining sauce-water mixture atop the cheese-egg mixture

Place four no-boil lasagna noodles atop the sauce-water mixture in a single layer

Spread remaining sauce-water mixture atop the no-boil lasagna noodles

Spread remaining Mozzarella cheese and Parmesan cheese atop the sauce-water mixture

three-layer lasagna product was prepared in a standard glass baking dish ( 13 by 9 by 2 in.) according to a recipe provided by the NPA (Table 2).

One inoculated lasagna per pathogenic organism was subjected to the baking treatment; each was prepared immediately before use. The inoculated lasagna was covered with tin foil and placed in an oven (preheated to $204^{\circ} \mathrm{C}$ ) for $50 \mathrm{~min}$. After baking, five replicate samples in total were collected from the lasagna from sample collection locations 1 through 5 (squares in Fig. 1); each replicate sample measured approximately 3 by 3 in. After collection, the inoculated noodles were separated from the rest of the lasagna in the replicate sample and were transferred to a sterile plastic bag. The inoculated noodles of the replicate sample were 


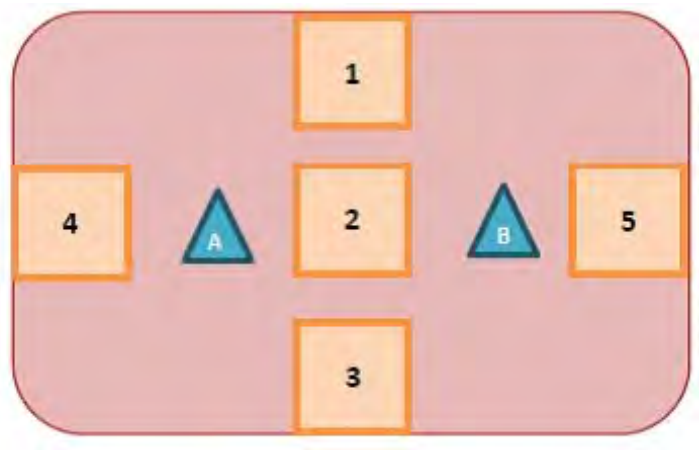

submerged in an ice bath for $20 \mathrm{~min}$ to cool the sample quickly and arrest further heat treatment. The sample was then diluted 1:10 (by weight) with PW. In addition, one uninoculated lasagna per pathogenic organism was assembled using uninoculated no-boil lasagna noodles and treated in the same manner as the inoculated lasagna; two replicate samples were collected from each uninoculated lasagna from two randomly selected sample collection locations.

One positive control lasagna per pathogenic organism was prepared. Each positive control lasagna was assembled using inoculated no-boil lasagna noodles according to the recipe provided by NPA (Table 2), but was proportionately scaled down in size and prepared in an appropriately sized pan. After preparation, the positive control lasagna was allowed to sit under ambient conditions (not baked) for $50 \mathrm{~min}$ to mimic the length of the baking treatment. After 50 min under ambient conditions, three replicate samples (measuring 3 by 3 in each) were collected from the positive control lasagna from randomly selected locations. The inoculated noodles were separated from the rest of the lasagna in the replicate sample and transferred to a sterile plastic bag. The inoculated noodles in the bag were then submerged in an ice bath for $20 \mathrm{~min}$ to mimic the handling of the baking-treated samples. The sample was then diluted 1:10 (by weight) with PW.

The water activity of the inoculated no-boil lasagna noodles was measured immediately before inclusion into the lasagna and immediately after the application of the baking cooking treatment. The temperature of each layer (top, middle, and bottom) of the inoculated lasagna was also measured in two locations (triangles in Fig. 1) after exposure to the baking treatment by using an $\mathrm{HH} 84$ rugged temperature data logger (OMEGA Engineering Inc.) and K type thermocouple (SA1XL-K-120-SRTC, OMEGA Engineering Inc.). The experiment was executed twice per pathogenic organism.

\section{Microbiological analyses}

Population levels of S. enterica and pathogenic E. coli were enumerated in each sample, as appropriate, by using a threetube most-probable-number (MPN) method adapted from Oyarzábal et al. (22). In brief, each sample was homogenized for $2 \mathrm{~min}$ and then serially diluted with PW. Appropriate
FIGURE 1. Sampling scheme for the inoculated and uninoculated lasagnas. Temperature measurement locations are depicted by the two blue triangles labeled "A" and "B". Sample collection locations for each replicate sample are indicated by the orange squares labeled 1, 2, 3, 4, and 5 . dilutions were then added to tubes of $10 \mathrm{~mL}$ of universal preenrichment broth (Accumedia), and the tubes were incubated at $35^{\circ} \mathrm{C}$ for 3 days. After incubation, turbid tubes from $S$. enterica-inoculated samples were streaked onto xylose lysine desoxycholate agar (Accumedia), and turbid tubes from pathogenic $E$. coli-inoculated samples were streaked onto sorbitol MacConkey agar (Accumedia) to confirm the presence of the pathogenic organisms. The xylose lysine desoxycholate agar and sorbitol MacConkey agar plates were incubated at $35^{\circ} \mathrm{C}$ for 1 day. A tube was considered "positive" if colonies exhibiting morphology and biochemical reactions typical to $S$. enterica on xylose lysine desoxycholate agar or E. coli on sorbitol MacConkey agar were observed; no additional confirmations were done. The limit of detection via $\mathrm{MPN}$ method was $0.5 \log \mathrm{MPN} / \mathrm{g}$.

\section{RESULTS AND DISCUSSION}

In the current study, the average population levels of S. enterica and pathogenic E. coli in the dry, inoculated elbow noodles, flat noodles, and no-boil lasagna noodles were all $>6.5 \log \mathrm{MPN} / \mathrm{g}$. All three cooking methods evaluated reduced $S$. enterica and pathogenic E. coli population levels to below the limit of detection, resulting in $>6$-log reductions (Table 3). At the time of this study, comparable studies investigating the lethality of common cooking treatments on S. enterica and pathogenic E. coli in pasta were not found. Therefore, the fate of these two pathogens in matrices with similar characteristics is discussed.

The heat resistance of microorganisms is greatly impacted by the water activity of the matrix and, in general, the heat resistance of $S$. enterica and $E$. coli decreases as water activity increases $(1,9,15,24,25,28,29)$. During cooking, the water activity of pasta transitions from low to high as the noodles become hydrated. Currently, it is not feasible or practical to define and monitor the rate at which the water activity of noodles increases during cooking. Thus, the water activity of pasta at the beginning and end of cooking must be considered when evaluating the lethality of a cooking process.

In this study, the water activity of the dry inoculated elbow noodles, flat noodles, and no-boil lasagna noodles ranged from 0.542 to 0.654 (Table 4). In low-water-activity matrices, 


\section{TABLE 3. Average population levels of S. enterica and pathogenic E. coll in inoculated pastas before and after the cooking treatments}

\begin{tabular}{|c|c|c|c|c|c|c|}
\hline \multirow{3}{*}{ Pathogenic organism } & \multicolumn{6}{|c|}{ Average population level $(\log \mathrm{MPN} / \mathrm{g})$ for each pasta type } \\
\hline & \multicolumn{2}{|c|}{ Elbow noodles } & \multicolumn{2}{|c|}{ Flat noodles } & \multicolumn{2}{|c|}{ No-boil lasagna noodles } \\
\hline & Trial 1 & Trial 2 & Trial 1 & Trial 2 & Trial 1 & Trial 2 \\
\hline \multicolumn{7}{|l|}{ S. enterica } \\
\hline Before treatment ${ }^{a}$ & $7.1 \pm 0.3$ & $7.4 \pm 0.0$ & $7.4 \pm 0.0$ & $7.3 \pm 0.2$ & $7.2 \pm 0.2$ & $7.2 \pm 0.2$ \\
\hline After treatment ${ }^{b}$ & $<0.5$ & $<0.5$ & $<0.5$ & $<0.5$ & $<0.5$ & $<0.5$ \\
\hline \multicolumn{7}{|l|}{ Pathogenic E. coli } \\
\hline Before treatment $^{a}$ & $6.9 \pm 0.2$ & $7.4 \pm 0.3$ & $7.2 \pm 0.5$ & $7.9 \pm 0.5$ & $6.9 \pm 0.4$ & $8.1 \pm 0.3$ \\
\hline After treatment ${ }^{b}$ & $<0.5$ & $<0.5$ & $<0.5$ & $<0.5$ & $<0.5$ & $<0.5$ \\
\hline $\begin{array}{l}{ }^{a} \operatorname{Avg} \pm \mathrm{SD}, n=3 \\
{ }^{b} \mathrm{Avg} \pm \mathrm{SD}, n=5\end{array}$ & & & & & & \\
\hline
\end{tabular}

\section{TABLE 4. Average water activity of S. enterica and pathogenic E. coli-inoculated pastas before and after cooking treatments}

\begin{tabular}{|c|c|c|c|c|c|c|}
\hline \multirow{3}{*}{ Pathogenic organism } & \multicolumn{6}{|c|}{ Average water activity for each pasta type } \\
\hline & \multicolumn{2}{|c|}{ Elbow noodles } & \multicolumn{2}{|c|}{ Flat noodles } & \multicolumn{2}{|c|}{ No-boil lasagna noodles } \\
\hline & Trial 1 & Trial 2 & Trial 1 & Trial 2 & Trial 1 & Trial 2 \\
\hline \multicolumn{7}{|l|}{ S. enterica } \\
\hline Before treatment ${ }^{a}$ & 0.542 & 0.567 & 0.653 & 0.596 & 0.561 & 0.562 \\
\hline After treatment ${ }^{a}$ & 0.996 & 0.994 & 1.003 & 1.002 & 0.990 & 0.985 \\
\hline \multicolumn{7}{|l|}{ Pathogenic E. coli } \\
\hline Before treatment ${ }^{a}$ & 0.585 & 0.583 & 0.576 & 0.590 & 0.557 & 0.598 \\
\hline After treatment ${ }^{a}$ & 0.989 & 0.988 & 0.990 & 0.992 & 0.979 & 0.971 \\
\hline
\end{tabular}

the heat resistance of $S$. enterica and pathogenic E. coli is expected to be extremely high. Archer et al. (2) determined the $D$-value of $S$. enterica serovar Weltevreden in wheat flour (water activity ranging from 0.50 to 0.60 ) to be 40 to 45 min at 75 to $77^{\circ} \mathrm{C}\left(\mathrm{z}\right.$-value of $\left.30.3^{\circ} \mathrm{C}\right)$. In addition, a study by Daryaei et al. (12) investigated the heat resistance of 22 strains of Shiga toxin-producing E. coli (both O157:H7 and non-O157 strains) in wheat flour ( $8 \%$ moisture content) and found that for 16 of the 22 strains tested, a $<2$-log reduction was observed after a heat treatment of $82^{\circ} \mathrm{C}$ for $5 \mathrm{~min}$. In a study by Suehr et al. (27), the D-value for E. coli O121 (isolated from the 2016 General Mills flour recall-outbreak) at $80^{\circ} \mathrm{C}$ was determined to be $4.58 \pm 0.40 \mathrm{~min}$ ( $z$-value of
$14.57 \pm 2.21^{\circ} \mathrm{C}$ ) in wheat flour, with a water activity of 0.45 . These studies indicate that the time required to observe adequate and substantial log reductions of $S$. enterica and pathogenic E. coli in dry pasta is far in excess of the those of the cooking treatments evaluated in the current study: microwave cooking, $2.5 \mathrm{~min}$; stovetop cooking, $6 \mathrm{~min}$; and baking cooking, $50 \mathrm{~min}$. Therefore, considering the extreme heat resistance of S. enterica and pathogenic E. coli in low-water-activity matrices, it is reasonable to assume that a majority of the lethality observed in this study occurred after the water activity of the pasta products had increased substantially, that is, at the end of the cooking treatments. 
TABLE 5. Average temperature of lasagna prepared with S. enterica and pathogenic E. coll-inoculated no-boil lasagna noodles after baking treatment

\begin{tabular}{|c|c|c|}
\hline \multirow{2}{*}{ Pathogenic organism } & \multicolumn{2}{|c|}{ Average temperature $\left({ }^{\circ} \mathrm{C}\right)$ for each lasagna layer after baking treatment } \\
\hline & Trial 1 & Trial 2 \\
\hline \multicolumn{3}{|l|}{ S. enterica } \\
\hline Top layer ${ }^{a}$ & 73.3 & 77.1 \\
\hline${\text { Middle layer }{ }^{a}}$ & 85.3 & 85.5 \\
\hline Bottom layer ${ }^{a}$ & 92.2 & 94.1 \\
\hline \multicolumn{3}{|l|}{ Pathogenic E. coli } \\
\hline Top layer $^{a}$ & 78.6 & 83.9 \\
\hline${\text { Middle layer }{ }^{a}}$ & 84.7 & 95.0 \\
\hline Bottom layer ${ }^{a}$ & 96.1 & 96.4 \\
\hline
\end{tabular}

${ }^{a} n=2$ for each layer.

At the end of all three cooking treatments evaluated in this study, the water activity of the inoculated pasta products was $>0.97$ (Table 4). At this water activity, S. enterica and pathogenic E. coli are expected to be much less resistant to heat than in a low-water-activity matrix. Although the heat resistance of $S$. enterica and pathogenic $E$. coli is a function of the organism strain and the characteristics of the matrix, in general the relative heat resistance of these organisms in high-water-activity matrices is expected to be as follows: S. enterica Senftenberg 775W $>$ E. coli $>$ Salmonella species (not including S. enterica Senftenberg $775 W)(13,15,21,26)$. The relatively high heat resistance of $S$. enterica Senftenberg $775 \mathrm{~W}$ in high-water-activity matrices is an interesting phenomenon and was the primary reason this particular strain was included in our study.

A review of studies on the heat resistance of Salmonella spp. conducted by Doyle and Mazzotta (13) found that of the high-water-activity matrices assessed (which included eggs, milk and dairy, poultry, and other meats), the heat resistance of Salmonella spp. was greatest in liquid eggs and liquid egg yolks. A study by Humphrey et al. (17) found that $S$. enterica Senftenberg $775 \mathrm{~W}$ was comparatively more resistant in homogenized whole egg than five other S. enterica strains (Enteritidis and Typhimurium) that had been isolated from egg sources. Thus, the heat resistance of S. enterica Senftenberg $775 \mathrm{~W}$ in a liquid egg matrix may provide context when assessing the lethality at the end of the cooking treatments evaluated in the present study. The heat resistance of $S$. enterica Senftenberg 775W in liquid whole eggs has been quantified by Humphrey et al. (17) and Mañas et al. (19); the $D$-values determined in these studies at 63 to $64^{\circ} \mathrm{C}$ were $2.8 \mathrm{~min}$ (calculated $\mathrm{z}$-value of $8.2^{\circ} \mathrm{C}$ ) and $1.2 \mathrm{~min}$ (z-value of $5.2^{\circ} \mathrm{C}$ ), respectively. Considering the Humphrey et al. (17)
$D$-value as a worst-case scenario, a 5-log reduction of $S$. enterica Senftenberg $775 \mathrm{~W}$ is predicted in $<1 \mathrm{~s}$ at $88.3^{\circ} \mathrm{C}$. This suggests that a final temperature of $88.3^{\circ} \mathrm{C}$ or higher may be sufficient to deliver adequate reduction of $S$. enterica and pathogenic $E$. coli instantaneously in a high-water-activity matrix.

In our study, the average temperature after the application of the microwave treatment was $>88.3^{\circ} \mathrm{C}$ in each trial executed (data not shown). In addition, although the temperature after the application of the stovetop treatment was not measured, it may be surmised that the flat noodles had reached a temperature $>88.3^{\circ} \mathrm{C}$ in the boiling water by the end of the cooking treatment, when the water activity of the noodles was high. Both of these cooking treatments resulted in $>6-\log$ reductions of $S$. enterica and pathogenic E. coli; thus, these results are in agreement with the predicted lethality of the microwave and stovetop cooking treatments.

The average temperature of the inoculated lasagnas after the application of the baking treatment differed between layers (Table 5). In all trials executed, the temperature of the top layer of the inoculated lasagna was the lowest of the three, followed by the middle layer, and finally the bottom layer. The lowest average temperature of the top, middle, and bottom layer recorded was $73.3,84.7$, and $92.2^{\circ} \mathrm{C}$ respectively $(n=2)$. Although a $5-\log$ reduction of $S$. enterica Senftenberg $775 \mathrm{~W}$ is predicted by Humphrey et al. (17) to be instantaneous at temperatures $>88.3^{\circ} \mathrm{C}$, a 5-log reduction at $73.3^{\circ} \mathrm{C}$ is expected to occur within $62 \mathrm{~s}$ (i.e., the top layer). The observance of $>6-\log$ reductions of $S$. enterica and pathogenic E. coli when the three layers of lasagna were evaluated as one sample indicates that the baking treatment was capable of delivering adequate reductions of these two pathogens. It is reasonable to assume that, considering the relatively long length of the baking treatment (i.e., $50 \mathrm{~min}$ ), 
the temperature of the no-boil lasagna noodles was $\geq 73.3^{\circ} \mathrm{C}$ for at least $62 \mathrm{~s}$ after hydration. It is also possible that $S$. enterica and pathogenic E. coli are not as heat resistant in a no-boil lasagna noodle matrix as $S$. enterica Senftenberg $775 \mathrm{~W}$ is in a liquid egg matrix, thus making the predictions based on the Humphrey et al. (17) model conservative.

\section{CONCLUSIONS AND RECOMMENDATIONS}

The ability of $S$. enterica and pathogenic E. coli to persist in low-water-activity products such as dry pasta for prolonged periods has prompted assessment of the microbiological lethality of cooking methods used to prepare dry pasta products for consumption. In this study, the lethality of three common cooking methods-microwave cooking of elbow noodles, stovetop cooking of flat noodles, and baking of no-boil lasagna noodles as part of lasagna-was assessed. The results indicated that these cooking methods delivered adequate and substantial log reductions of $S$. enterica and pathogenic E. coli, demonstrating that the cooking processes evaluated are capable of mitigating the microbiological food safety risks associated with dry pasta.

Recommendations for future research in this area may include evaluating shorter cooking times, investigating the lethality of the cooking methods for different pasta types, and assessing the lethality of nontraditional cooking methods (based on innovative consumer behavior).

\section{ACKNOWLEDGMENTS}

The authors acknowledge the NPA for technical and financial support and the contributions of Alexis FreierJohnson (8th Avenue Food \& Provisions), Al Lucia and Joe DeFrancisci (DEMACO, De Francisci Machine Company), Maile G. Hermida (Hogan Lovells US LLP [legal counsel]), Gary River (A. Zerega's Sons, Inc.), and Mike Hoar (Riviana Foods, Inc.). In addition, the authors thank Arthur Velador, Daljit Kaur, Fue Thao, Javier Navarro, Jennifer Lee, Justine Angulo, Mary Jane Aquisap, Meina He, and Mirela Stiuca for work in the Eurofins lab.

\section{REFERENCES}

1. Aljarallah, K. M., and M. R. Adams. 2007. Mechanisms of heat inactivation in Salmonella serotype Typhimurium as affected by low water activity at different temperatures. J. Appl. Microbiol. 102:153-160.

2. Archer, J., E. T. Jervis, J. Bird, and J. E. Gaze. 1998. Heat resistance of Salmonella Weltevreden in low-moisture environments. J. Food Prot. 61:969-973.

3. Blessington, T., E. J. Mitcham, and L. J. Harris. 2014. Growth and survival of Enterobacteriaceae and inoculated Salmonella on walnut hulls and maturing walnut fruit. J. Food Prot. 77:1462-1470.

4. Centers for Disease Control and Prevention. 1998. Multistate outbreak of Salmonella serotype Agona infections linked to toasted oats cereal - United States, April-May, 1998. Morb. Mortal. Wkly. Rep. 47:462-464.

5. Centers for Disease Control and Prevention. 2008. Multistate outbreak of Salmonella Agona infections lined to rice and wheat puff cereal (FINAL UPDATE). Available at: https://www.cdc.gov/salmonella/2008/ricewheat-puff-cereal-5-13-2008.html. Accessed 12 October 2019.

6. Centers for Disease Control and Prevention. 2014. Multistate outbreak of Salmonella infections linked to organic sprouted chia powder (FINAL UPDATE). Available at: http:// www.fda.gov/Safety/Recalls/ucm 423223. htm. Accessed 14 January 2016.

7. Centers for Disease Control and Preventation. 2016. Multistate outbreak of shiga toxin-producing Escherichia coli infections linked to flour. Available at: https://www.cdc.gov/ ecoli/2016/o121-06-16/. Accessed 9 September 2016.
8. Centers for Disease Control and Prevention. 2019. Outbreak of E. coli infections linked to flour. Available at: https://www.cdc.gov/ ecoli/2019/flour-05-19/index.html. Accessed 2 December 2019.

9. Chiewchan, N., W. Pakdee, and S. Devahastin 2007. Effect of water activity on thermal resistance of Salmonella Krefeld in liquid medium and on rawhide surface. Int. J. Food Microbiol. 114:43-49.

10. Danyluk, M. D., A. R. Uesugi, and L. J. Harris. 2005. Survival of Salmonella Enteritidis PT 30 on inoculated almonds after commercial fumigation with propylene oxide. J. Food Prot. 68:1613-1622.

11. Daryaei, H., W. Peñaloza, I. Hildebrandt, K. Krishnamurthy, P. Thiruvengadam, and J. Wan. 2017. Heat inactivation of Shiga toxin-producing Escherichia coli in a selection of low moisture foods. Food Control 85:48-56.

12. Daryaei, H., Q. Sui, H. Liu, A. Rehkopf, W. Peñaloza, A. Rytz, Y. Luo, and J. Wan. 2020. Heat resistance of Shiga toxin-producing Escherichia coli and potential surrogates in wheat flour at two moisture levels. Food Control 108:106788.

13. Doyle, M. E., and A. S. Mazzotta. 2000. Review of studies on the thermal resistance of salmonellae. J. Food Prot. 63:779-795.

14. Food Safety News. 2015. Navajo Pride flour recalled for possible Salmonella Contamination. Available at: http://www. foodsafetynews.com/2015/04/navajo?pride?flour? recalled?for?possible?salmsalmon?contamination/\#.VtnZOPkrLIV. Accessed 15 January 2016.

15. Geopfert, J. M., I. K. Iskander, and C. H. Amundson. 1970. Relation of the heat resistance of salmonellae to the water activity of the environment. Appl. Microbiol. 19:429-433.
16. He, Y., D. Guo, J. Yang, M. Lou Tortorello, and W. Zhang. 2011. Survival and heat resistance of Salmonella enterica and Escherichia coli O157:H7 in peanut butter. Appl. Environ. Microbiol. 77:8434-8438.

17. Humphrey, T. J., P. A. Chapman, B. Rowe, and R. J. Gilbert. 1990. A comparative study of the heat resistance of salmonellas in homogenized whole egg, egg yolk or albumen. Epidemiol. Infect. 104:237-241.

18. Kimber, M. A., H. Kaur, L. Wang, M. D. Danyluk, and L. J. Harris. 2012. Survival of Salmonella, Escherichia coli O157:H7, and Listeria monocytogenes on inoculated almonds and pistachios stored at $-19,4$, and $24^{\circ} \mathrm{C}$. J. Food Prot. 75:1394-1403.

19. Mañas, P., R. Pagán, I. Alvarez, and S. Condón Usón. 2003. Survival of Salmonella Senftenberg $775 \mathrm{~W}$ to current liquid whole egg pasteurization treatments. Food Microbiol. 20:593-600.

20. McCallum, L., S. Paine, K. Sexton, M. Dufour, K. Dyet, M. Wilson, D. Campbell, D. Bandaranayake, and V. Hope. 2013. An outbreak of Salmonella Typhimurium phage type 42 associated with the consumption of raw flour. Foodborne Pathog. Dis. 10:159-164.

21. Ng, H., H. G. Bayne, and J. A. Garibaldi. 1969. Heat resistance of Salmonella: the uniqueness of Salmonella Senftenberg 775W. Appl. Microbiol. 17:78-82.

22. Oyarzábal, O. A., M. C. L. Nogueira, and D. E. Gombas. 2003. Survival of Escherichia coli O157:H7, Listeria monocytogenes, and Salmonella in juice concentrates. J. Food Prot. 66:1595-1598. 
23. Podolak, R., E. Enache, W. Stone, D. G. Black, and P. H. Elliott. 2010. Sources and risk factors for contamination, survival, persistence, and heat resistance of Salmonella in low-moisture foods. J. Food Prot. 73:1919-1936.

24. Rachon, G., W. Peñaloza, and P. A. Gibbs. 2016. Inactivation of Salmonella, Listeria monocytogenes and Enterococcus faecium NRRL B-2354 in a selection of low moisture foods. Int. J. Food Microbiol. 231:16-25.

25. Santillana Farakos, S. M., J. F. Frank, and D. W. Schaffner. 2013. Modeling the influence of temperature, water activity and water mobility on the persistence of Salmonella in low-moisture foods. Int. J. Food Microbiol. 166:280-293.

26. Sörqvist, S. 2003. Heat resistance in liquids of Enterococcus spp., Listeria spp., Escherichia coli, Yersinia enterocolitica, Salmonella spp. and Campylobacter spp. Acta Vet. Scand. 44:1-19.

27. Suehr, Q. J., N. M. Anderson, and S. E. Keller. 2019. Desiccation and thermal resistance of Escherichia coli O121 in wheat flour. J. Food Prot. 82:1308-1313.

28. Sumner, S., T. Sandros, M. Harmon, V. Scott, and D. Bernard. 1991. Heat resistance of Salmonella Typhimurium and Listeria monocytogenes in sucrose solutions of various water activities. J. Food Sci. 56:1741-1743.
29. Syamaladevi, R. M., J. Tang, R. Villa-Rojas, S. Sablani, B. Carter, and G. Campbell. 2016. Influence of water activity on thermal resistance of microorganisms in low-moisture foods: a review. Compr. Rev. Food Sci. Food Saf. 15:353-370.

30. U.S. Food and Drug Administration. 2011. Recall - firm press release: thumb oilseed recalls soy flour (utilized to manufacture human and animal food) due to Salmonella contamination. Available at: http://www. fda.gov/Safety/Recalls/ucm274402.htm. Accessed 14 January 2016.

31. U.S. Food and Drug Administration. 2012 Bad Bug Book: Handbook of Foodborne Pathogenic Microorganisms and Natural Toxins. Available at: http://www.fda.gov/ downloads/Food/FoodborneIllnessContaminants/UCM297627.pdf. Accessed 1 January 2016.

32. U.S. Food and Drug Administration. 2014. Lundberg Family Farms recalls eco-farmed and organic brown rice flour because of possible health risk. Available at: http://www. fda.gov/Safety/Recalls/ucm423223.htm. Accessed 21 January 2016.
33. U.S. Food and Drug Administration. 2017. Smucker Foods of Canada Corp. announces a voluntary recall on Select Golden Temple ${ }^{\varpi}$, $\mathrm{Swad}^{\circledR}$, and Maya ${ }^{\circledR}$ flour product in U.S. due to possible E. coli $\mathrm{O} 121$ contamination. Available at: https://www.fda.gov/safety/ recalls-market-withdrawals-safety-alerts/ smucker-foods-canada-corp-announces-voluntary-recall-select-golden-templer-swadr-and-mayar-flour\#: :text=today announced a voluntary recall,coli 012 contamination.\&text=Impacted product. Accessed 12 July 2020.

34. U.S. Food and Drug Administration. 2019 General Mills recalls five pound bags of Gold Medal unbleached flour. Available at: https:// www.fda.gov/safety/recalls-market-withdrawals-safety-alerts/general-mills-recallsfive-pound-bags-gold-medal-unbleached-allpurpose-flour. Accessed 12 February 2019. 